\title{
Quel est le degré de décentralisation des systèmes d'éducation et son incidence pour les établissements d'enseignement?
}

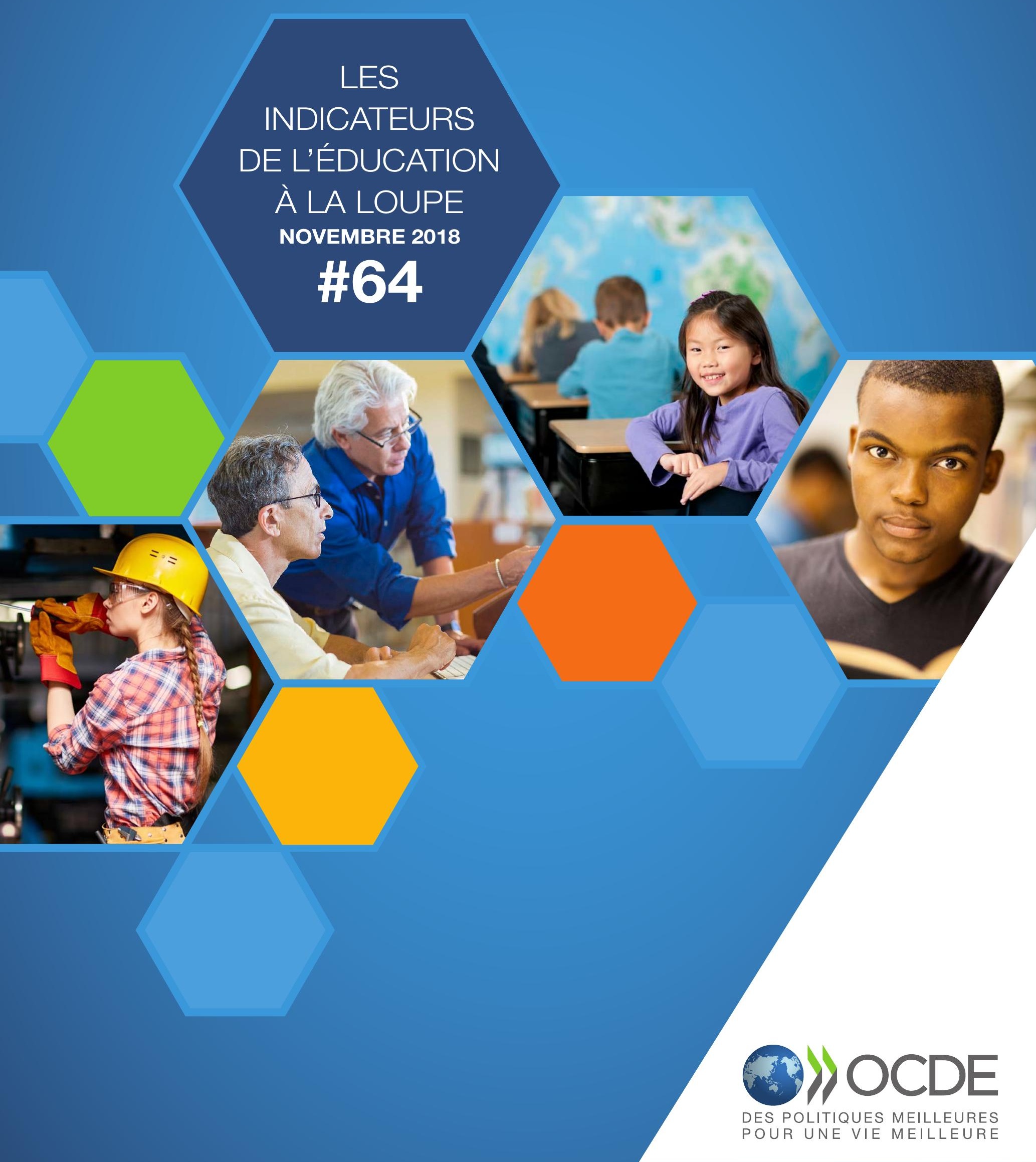




\section{Quel est le degré de décentralisation des systèmes d'éducation et son incidence pour les établissements d'enseignement?}

- Depuis le début des années 80, l'autonomie des établissements d'enseignement s'est considérablement accrue, mais le degré d'autonomie et les domaines de décision où elle s'exerce varient sensiblement entre les pays.

- Parallèlement au développement de l'autonomie des établissements d'enseignement, le rôle des exécutifs centraux s'est aussi renforcé dans la définition des normes, programmes scolaires et évaluations.

- Au sein des établissements d'enseignement, la répartition des responsabilités entre les différents acteurs varie aussi en fonction des pays et des domaines de décision.

- L'octroi d'un plus grand pouvoir décisionnel aux établissements d'enseignement peut avoir un effet positif sur les résultats d'apprentissage lorsqu'il est utilisé à bon escient. Toutefois, la difficulté et la complexité du rôle des chefs d'établissement s'en trouvent aussi accentuées, avec une augmentation de leur charge de travail.

\section{Encadré 1. Sources des données et définitions}

Ce numéro des Indicateurs de l'éducation à la loupe s'appuie sur l'important corpus d'analyses déjà réalisées sur les degrés de décentralisation des systèmes d'éducation et d'autonomie des établissements d'enseignement:

- Regards sur l'éducation 2018 (OCDE, 2018[1]) présente des données sur les niveaux de prise de décision dans l'enseignement public du premier cycle du secondaire, avec une ventilation par niveau de pouvoir de décision (exécutif central, exécutif des entités fédérées, exécutif local, établissements d'enseignement ou conseils/comités de direction des établissements d'enseignement, niveaux multiples) et par modalité de prise de décision (degré d'autonomie ou de " partage " - en toute autonomie, après consultation d'instances situées à d'autres niveau du système d'éducation, en toute indépendance mais dans le respect d'un cadre défini par un niveau supérieur de l'exécutif, ou autre). Les données couvrent 23 types de décisions, regroupées en quatre grands domaines :

- organisation de l'enseignement (par exemple, admission des élèves, temps d'instruction, regroupement des élèves)

- gestion du personnel (par exemple, embauche et licenciement, fonctions et conditions de travail, barèmes salariaux du personnel enseignants et des chefs d'établissement)

- planification et structures (par exemple, conception des programmes de cours, choix des matières enseignées dans chaque établissement, définition du contenu des cours)

- gestion des ressources (par exemple, affectation et utilisation des ressources au titre du personnel enseignant et des chefs d'établissement).

Chaque domaine de décision est pondéré afin d'équilibrer le poids de chacun des quatre domaines.

- Le Programme international pour le suivi des acquis des élèves (PISA) 2015 (OCDE, 2016[2]) présente des données sur les réponses des chefs d'établissements scolarisant des jeunes de 15 ans (établissements publics et privés, des premier et deuxième cycles du secondaire) à son questionnaire d'enquête.

- L'Enquête internationale de l'OCDE sur l'enseignement et l'apprentissage (TALIS) 2013 (OCDE, 2014[3]) présente des données sur les réponses des chefs d'établissement du premier cycle du secondaire (établissements publics et privés) à son questionnaire d'enquête, notamment sur la mesure dans laquelle la responsabilité des prises de décisions dans différents domaines incombe aux établissements d'enseignement, et non à l'exécutif local ou national.

\section{Quelle est la répartition du pouvoir décisionnel au sein du système d'éducation?}

Depuis le début des années 80, plusieurs pays, dont l'Australie, le Canada, la Finlande, les Pays-Bas, le Royaume-Uni et la Suède, ont entrepris des réformes systémiques afin de décentraliser le pouvoir décisionnel vers les exécutifs locaux et les établissements d'enseignement. Ces derniers se sont ainsi vus accorder une plus grande autonomie pour les prises de décisions concernant les programmes scolaires et l'affectation des ressources humaines et financières. Cela reposait sur l'hypothèse que les acteurs locaux et les établissements d'enseignement semblaient les mieux placés pour comprendre les besoins des communautés locales et des élèves, et prendre ainsi des décisions plus éclairées pour la gestion des ressources (OCDE, 2016 ${ }_{[2]}$; Hanushek, Link et Woessmann, 2012 $2_{[4]}$ ). 


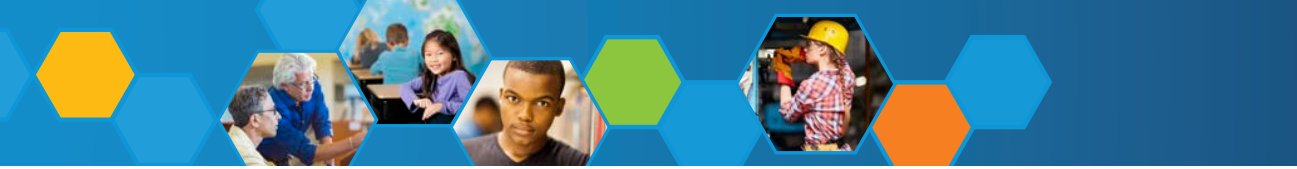

Dans les pays de l'OCDE, le pourcentage de décisions prises à chaque niveau de l'exécutif donne une première indication du degré de décentralisation des systèmes d'éducation. La majorité des décisions sont prises au niveau de l'exécutif central ou de celui des entités fédérées dans moins d'un tiers des pays et économies membres ou partenaires de l'OCDE, mais principalement au niveau de l'exécutif local ou des établissements d'enseignement dans environ la moitié de ceux-ci. Le pourcentage de décisions prises au niveau de l'exécutif local ou des établissements d'enseignement varie toutefois sensiblement : de $8 \%$ en Turquie, à plus de $90 \%$ en Écosse (RU), aux Pays-Bas et en République tchèque. Dans d'autres pays tels que le Danemark et le Japon, les décisions sont réparties entre plusieurs niveaux de l'exécutif (graphique 1).

GRAPHIQUE 1 / Pourcentage de décisions prises à chaque niveau de l'exécutif dans l'enseignement public du premier cycle du secondaire (2017)

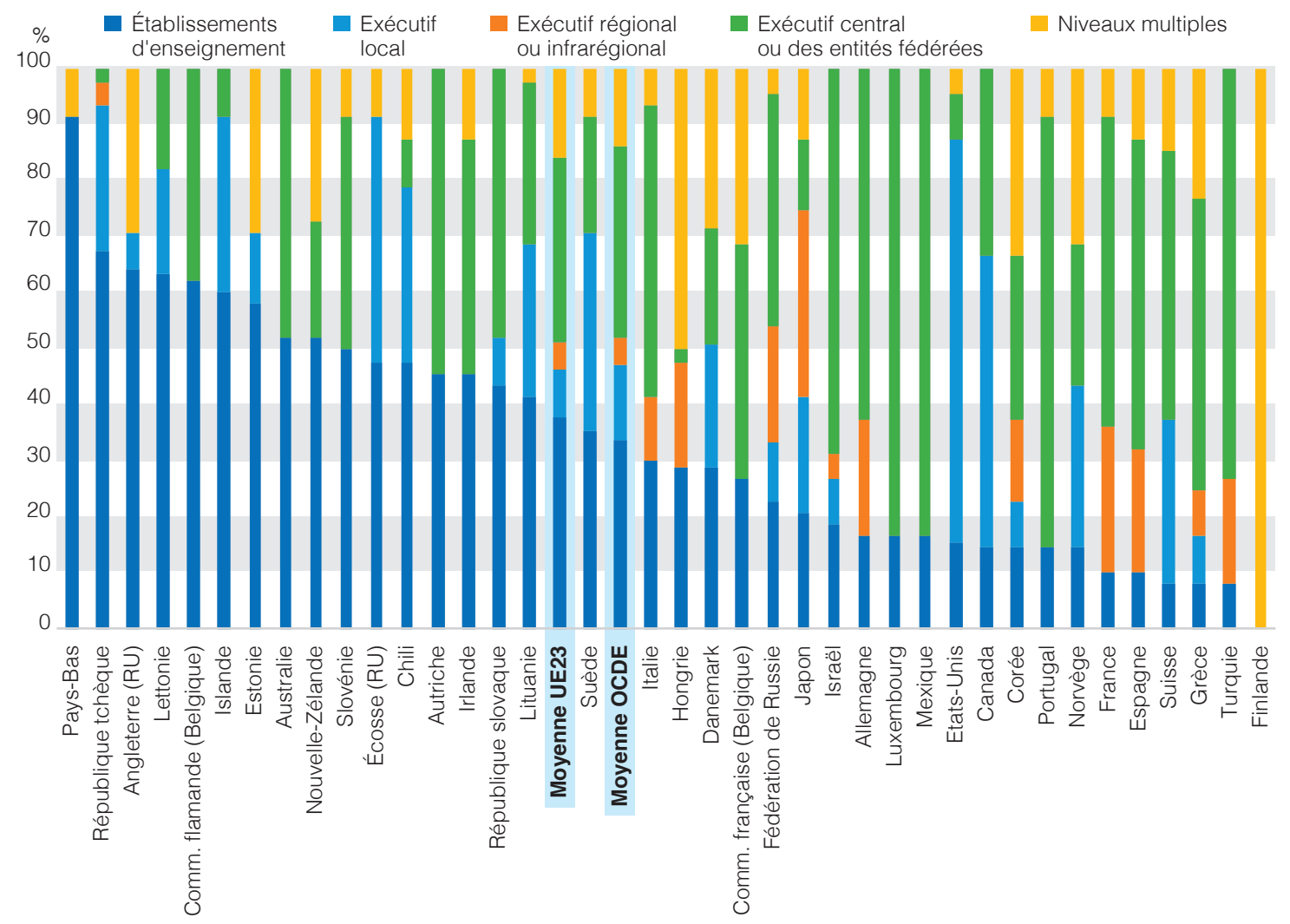

Les pays sont classés par ordre décroissant du pourcentage de décisions prises au niveau des établissements d'enseignement. Source: OCDE (2018), tableau D6.1. Voir les notes à l'annexe 3 (http://dx.doi.org/10.1787/eag-2018-36-fr).

Le niveau des prises de décisions et le degré d'autonomie dépendent aussi du type ou du domaine de décision. La comparaison des domaines met au jour des différences significatives entre les pays et au sein de ceux-ci. En moyenne, dans les pays et économies de l'OCDE, les établissements d'enseignement ou les exécutifs locaux prennent $63 \%$ des décisions relatives à l'organisation de l'enseignement, dont environ $20 \%$ seulement en toute autonomie. Aux Pays-Bas et en République tchèque, les établissements d'enseignement prennent toutes les décisions relatives à l'organisation de l'enseignement, mais principalement dans le respect d'un cadre défini par un niveau supérieur de l'exécutif. Les décisions relatives à la planification et aux structures, ainsi qu'à la gestion du personnel, sont en général plus centralisées : en moyenne, environ 50\% de celles relatives à la planification et aux structures, et 35\% de celles relatives à la gestion du personnel, sont prises au niveau de l'exécutif central ou de celui des entités fédérées. Même lorsque ces décisions sont décentralisées vers les exécutifs locaux ou les établissements d'enseignement, elles sont rarement prises en toute autonomie. Le niveau décisionnel varie en outre nettement entre les décisions relatives à l'affectation des ressources et celles relatives à leur utilisation. Bien que les exécutifs centraux ou ceux des entités fédérées décident de l'affectation des ressources dans près de la moitié des pays et économies, les décisions relatives à leur utilisation au sein des établissements d'enseignement au titre du personnel et de la formation continue des enseignants et des chefs d'établissement relèvent plus souvent des établissements d'enseignement ou des exécutifs locaux (OCDE, 2018 11 ). Dans l'ensemble, environ la moitié des décisions relatives à la gestion des ressources prises au niveau des établissements d'enseignement le sont en toute autonomie (graphique 2).

\section{Quel rôle les niveau supérieurs de l'exécutif jouent-ils dans les systèmes décentralisés?}

Parallèlement au développement de l'autonomie des établissements d'enseignement, le rôle des exécutifs centraux s'est aussi accentué dans la définition des normes, programmes scolaires et évaluations, renforçant ainsi les mécanismes de responsabilisation des établissements d'enseignement. Dans l'enseignement public du premier cycle du secondaire, un tiers environ seulement des décisions prises au niveau des établissements d'enseignement 


\section{GRAPHIQUE 2 / Pourcentage de décisions prises au niveau de l'exécutif local ou des établissements d'enseignement dans l'enseignement public du premier cycle du secondaire, selon la modalité et le domaine de décision (pays de l'OCDE) (2017)}

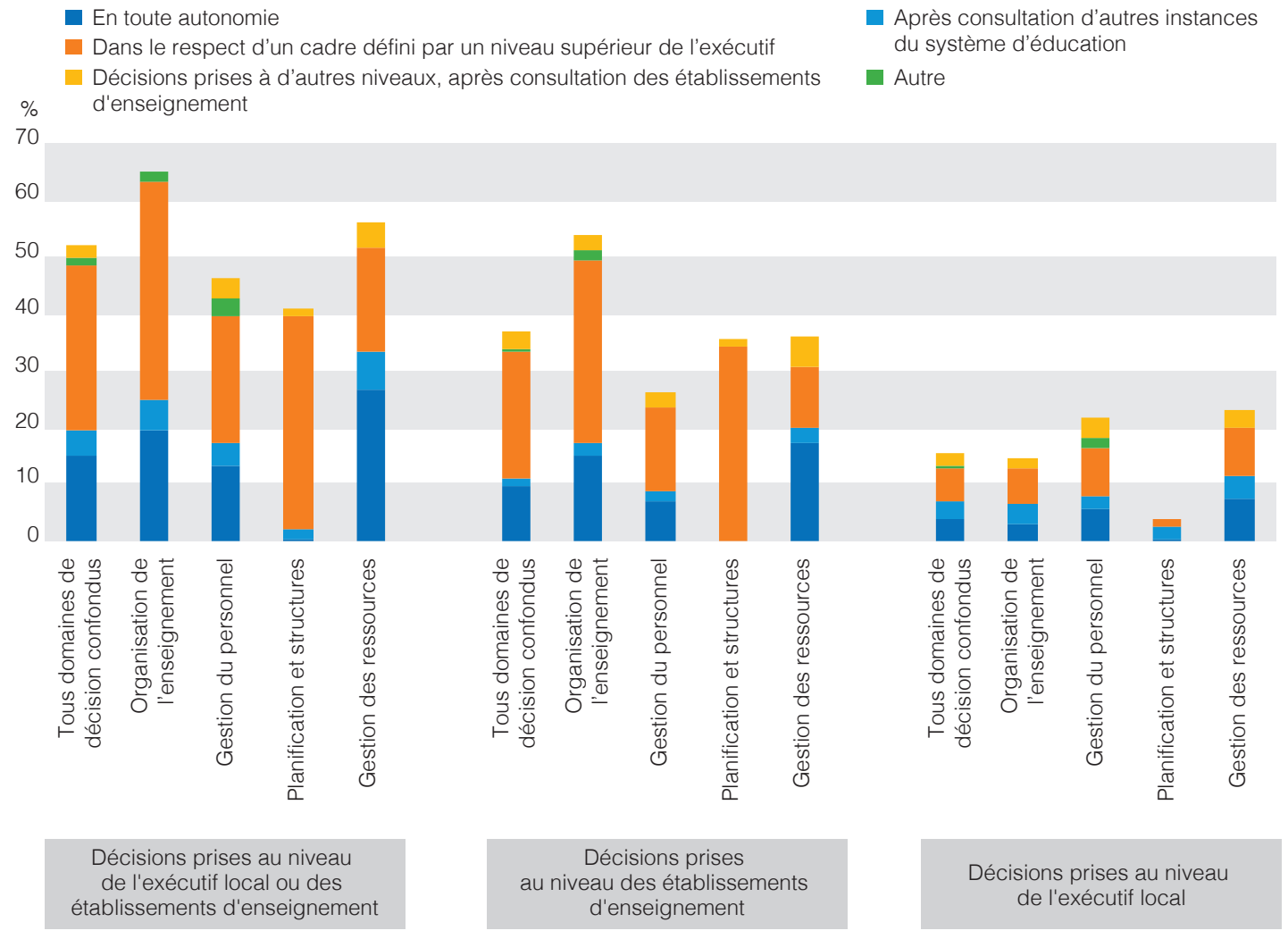

Source : OCDE (2018), tableau D6.3 et tableaux D6.4a, b et c disponibles en ligne. Voir les notes à l'annexe 3 (http://dx.doi.org/10.1787/eag-2018-36-fr).

ou des exécutifs locaux le sont en toute autonomie, contre environ deux tiers dans le respect d'un cadre défini par un niveau supérieur de l'exécutif (OCDE, 2018 $8_{[1]}$ ).

Le rôle de l'exécutif central est de garantir l'équité et la responsabilisation du système d'éducation, en particulier lorsque le renforcement de l'autonomie des établissements d'enseignement se conjugue avec la liberté, pour les parents, de choisir l'établissement de leur enfant. Plusieurs systemes, tels que la communauté flamande de Belgique, le Royaume-Uni et la Suède, ont encouragé une combinaison de libre choix de l'établissement d'enseignement et de renforcement de l'autonomie. Ce type de politiques entend permettre aux parents de scolariser leur enfant dans l'établissement de leur choix, que ce soit dans l'enseignement public ou privé. L'autonomie des établissements d'enseignement et le libre choix des parents pourraient pallier certaines inadéquations entre l'offre éducative et la demande des parents, renforcer la concurrence entre les établissements et donc stimuler l'innovation.

La frontière entre différenciation et ségrégation scolaire est toutefois ténue. Conjuguée au libre choix des parents, l'autonomie des établissements d'enseignement peut aussi entraîner l'isolement et la ségrégation des établissements : si certains d'entre eux réussiront à tirer profit d'une plus grande autonomie, d'autres pourront néanmoins ne pas parvenir à gérer ces responsabilités supplémentaires. Et si les parents issus de milieux socio-économiques plus favorisés ont les moyens de choisir le meilleur établissement pour leur enfant, ceux de milieux plus défavorisés peuvent avoir à privilégier des critères plus financiers que qualitatifs lors du choix de l'établissement de leur enfant, et être moins bien informés que les parents les plus favorisés (OCDE, 2016 [ OCDE, 2017 $[5]$; Rodriguez et Hovde, 2002 $\left.2_{[6]}\right)$. Cependant, si le risque de ségrégation existe bel et bien, les données comparatives internationales ne mettent au jour aucune relation entre la prévalence de la liberté de choix de l'établissement d'enseignement et la ségrégation, qui dépend principalement des conditions sous-tendant ce choix (OCDE, 2017 ${ }_{[51}$ ). Il est donc essentiel pour l'exécutif central de maintenir une vision stratégique et des directives claires afin de veiller à ce que le développement du libre choix de l'établissement d'enseignement ne se fasse pas au détriment de l'équité de l'offre éducative.

\section{Quelle est la répartition du pouvoir décisionnel au sein des établissements d'enseignement?}

Différents acteurs peuvent intervenir dans les décisions prises au niveau des établissements d'enseignement. Les chefs d'établissement sont souvent considérés comme l'autorité en charge du développement et de la gestion de l'établissement, assurant le lien entre enseignants, parents et niveau supérieurs du système d'éducation. L'offre d'une éducation de qualité requiert toutefois un esprit de collaboration et le partage des décisions entre les différents acteurs (Burns et Köster, 2016 $6_{[7]}$; Cheng, Ko et Lee, 2016 $6_{[8]}$ ). 
Le partage des responsabilités entre enseignants et chefs d'établissement dépend en grande partie de la capacité et de la volonté de ces derniers d'accorder un pouvoir de décision aux enseignants. Il dépend également de l'existence d'une formation adéquate destinée aux uns et aux autres qui vise à les doter des outils dont ils ont besoin pour assumer leurs responsabilités supplémentaires. Dans les pays de l'OCDE, la Lettonie, le Portugal, la Suède et la Turquie sont les seuls pays à disposer d'une législation stipulant le devoir de participation des enseignants aux activités de l'établissement (qu'elles soient de gestion ou autres). À l'inverse, la participation des enseignants aux activités de gestion est à la discrétion de chaque établissement d'enseignement dans 19 des 38 pays et économies à l'étude (OCDE, 2018 $\left.{ }_{[1]}\right)$. En moyenne, dans les pays de l'OCDE, $72 \%$ des chefs d'établissement indiquent donner aux enseignants la possibilité d'intervenir dans les décisions concernant l'établissement au moins une fois par mois, et 73\%, les inciter à instaurer une culture de l'établissement axée sur l'amélioration continue. En revanche, seuls $34 \%$ des chefs d'établissement déclarent demander aux enseignants d'évaluer les pratiques de gestion au moins une fois par mois, et 10\% environ indiquent ne jamais le faire. Cependant, en Corée, aux États-Unis et en Turquie, plus de $65 \%$ des élèves sont scolarisés dans des établissements où, selon le chef d'établissement, les enseignants sont invités à participer à l'évaluation des pratiques de gestion au moins une fois par mois (OCDE, $\left.2016_{[2]}\right)$.

Tout comme à l'échelle du système d'éducation, la répartition des responsabilités au sein des établissements d'enseignement dépend du domaine de prise de décision. Les chefs d'établissement prennent la majorité des décisions relatives à la gestion des ressources, en particulier du personnel. En moyenne, dans les pays de l'OCDE, $70 \%$ des élèves de 15 ans sont scolarisés dans des établissements où le chef d'établissement indique avoir une responsabilité significative concernant la sélection des enseignants, et $57 \%$ lorsque cette responsabilité concerne le renvoi des enseignants. Ces pourcentages dépassent $97 \%$ en Islande, en République slovaque et en République tchèque, mais sont inférieurs à 10\% en Grèce et en Turquie. À l'inverse, en moyenne dans les pays de l'OCDE, seuls $10 \%$ des élèves sont scolarisés dans des établissements où, selon le chef d'établissement, les enseignants ont une responsabilité significative concernant la sélection des enseignants, et 1\% lorsque cette responsabilité concerne le renvoi des enseignants. Les enseignants ont en général un pouvoir de décision sur les questions relatives aux programmes scolaires et aux évaluations des élèves. Dans les pays de l'OCDE, $82 \%$ des élèves sont scolarisés dans des établissements où les enseignants sont principalement en charge du choix des manuels scolaires, et $68 \%$, dans des établissements où les enseignants sont principalement en charge du choix du contenu des cours. Ces pourcentages varient toutefois considérablement entre les pays : de moins de $5 \%$ en Grèce, à $94 \%$ en NouvelleZélande (OCDE, 2016 $\left.{ }_{[9]}\right)$.

Les établissements d'enseignement peuvent aussi faire participer activement les parents au processus décisionnel. En moyenne, $96 \%$ des élèves sont scolarisés dans des établissements qui, selon le chef d'établissement, créent une ambiance conviviale et ouverte visant à favoriser l'implication des parents, et $77 \%$ dans des établissements où les parents interviennent dans les décisions concernant l'établissement. Dans les pays de l'OCDE, les parents d'enfants scolarisés dans des établissements d'enseignement privés participent davantage aux activités en lien avec l'établissement que ceux dont les enfants fréquentent un établissement public. Les parents restent des partenaires clés des enseignants et des chefs d'établissement, en assurant la transition entre la maison et l'école, et en jouant un rôle essentiel dans la création d'un environnement d'apprentissage positif et à l'écoute des besoins des élèves (OCDE, 2016 $6_{22}$. La législation sur la participation des parents varie toutefois sensiblement d'un pays à l'autre, et aucune corrélation n'a été établie entre le degré de leur pouvoir décisionnel et les résultats des élèves.

En revanche, dans les établissements où enseignants et chefs d'établissement collaborent plus étroitement à la gestion et au développement de l'établissement, les élèves tendent à obtenir de meilleurs résultats en sciences (OCDE, 2016 $[2]$ ). Ce constat vaut particulièrement lorsque les chefs d'établissement et les enseignants ont plus de latitude pour la conception des programmes scolaires et les dispositifs d'évaluation.

\section{Quelle est l'incidence de l'autonomie sur le fonctionnement et la direction des établissements d'enseignement?}

Le lien entre autonomie des établissements d'enseignement et performance des élèves a été largement débattu (Hanushek, Link et Woessmann, 2012 $\left.{ }_{[4}\right)$. Plus spécifiquement, lorsqu'elle se conjugue avec de solides dispositifs de responsabilisation, l'autonomie des établissements pour l'affectation des ressources est associée à une amélioration des résultats des élèves (OCDE, 2011 $1_{[10]}$; OCDE, 2016 $[2]$ ). Toutefois, l'impact que le renforcement de l'autonomie des établissements d'enseignement a sur leur fonctionnement - notamment sur les conditions de travail des chefs d'établissement et des enseignants, leurs relations mutuelles et le climat général de l'établissement - demeure mal compris.

Le renforcement de l'autonomie modifie le rôle des acteurs éducatifs. Les établissements d'enseignement exercent un plus grand contrôle sur leur utilisation des ressources et sont donc confrontés à une plus forte exigence de résultats. Les chefs d'établissement et les enseignants sont par exemple de plus en plus amenés à assumer tout un éventail de rôles exigeants, dont la gestion des ressources humaines et financières et l'encadrement pédagogique (Pont, Nusche et Moorman, 2008 $\left.{ }_{[11]}\right)$.

De nombreux chefs d'établissement se plaignent de l'importance de leur charge de travail, même si ceux qui pratiquent un niveau plus élevé de partage des responsabilités et d'encadrement pédagogique ${ }^{1}$ font part d'une plus grande satisfaction professionnelle (OCDE, $2014_{[3]}$. La multiplication des fonctions, l'inadéquation de la préparation et de la formation, la limitation des perspectives de carrière et l'insuffisance des soutiens sont autant de facteurs susceptibles d'accentuer la difficulté à attirer les candidats vers les postes de direction d'établissement (Pont, Nusche et Moorman, 2008 $\left.{ }_{[11]}\right]$. Si le système de soutien aux chefs d'établissement et aux autres acteurs locaux n'est pas adapté en conséquence, le renforcement de l'autonomie peut clairement entraîner une augmentation des niveaux de stress et l'apparition de défis considérables pour les établissements d'enseignement, éléments qui peuvent à leur tour affecter la performance des élèves. La baisse des résultats de la Suède dans l'enquête PISA suite à la mise en œuvre de sa réforme de décentralisation totale dans les années 90 s'explique ainsi en partie par le manque de préparation des différents acteurs à des changements de cette envergure (Blanchenay, Burns et Köster, 2014 $\left.4_{[12]}\right)$. Il est donc nécessaire d'adapter la formation et l'évaluation des chefs d'établissement et des enseignants, ainsi que le soutien qu'on leur apporte, afin de pouvoir faire face aux exigences croissantes accompagnant le renforcement de l'autonomie des établissements d'enseignement (OCDE, 2017 ${ }_{[5]}$ ).

1 Par encadrement pédagogique, on entend : favoriser la coopération entre les enseignants pour élaborer des pratiques pédagogiques innovantes; prendre des mesures concrètes pour que les enseignants s'investissent dans l'amélioration de leurs compétences pédagogiques; et prendre des mesures concrètes pour que les enseignants se sentent responsables des résultats de leurs élèves. 


\section{Pour conclure}

Le renforcement de l'autonomie des établissements d'enseignement est une mesure populaire, qui peut toutefois aussi s'avérer complexe et parfois même sujette à controverse. Nombre de systèmes d'éducation ont renforcé l'autonomie des établissements d'enseignement dans l'espoir de gagner en efficacité et de mieux répondre aux besoins locaux. Dans certains pays, toutefois, le renforcement de cette autonomie soumet les établissements d'enseignement et les acteurs locaux à une pression accrue. Pour porter ses fruits, le renforcement de l'autonomie des établissements d'enseignement doit se fonder sur un ensemble d'éléments clés : un cadre national solide et une vision stratégique claire ; des programmes de formation bien adaptés pour les chefs d'établissement et les enseignants ; de solides dispositifs de responsabilisation ; et l'instauration d'un climat de collaboration, tant entre les établissements d'enseignement qu'au sein de ceux-ci.

\section{POUR TOUT COMPLÉMENT D'INFORMATION :}

[12] Blanchenay, P., T. Burns et F. Köster (2014), « Shifting Responsibilities - 20 Years of Education Devolution in Sweden: A Governing Complex Education Systems Case Study », Documents de travail de l'OCDE sur l'éducation, n 104, Éditions OCDE, Paris, http://dx.doi.org/10.1787/5jz2jg1rqrd7-en.

[7] Burns, T. et F. Köster (2016), Governing Education in a Complex World, Le recherche et l'innovation dans l'enseignement, Éditions OCDE, Paris, https://doi.org/10.1787/9789264255364-en.

[8] Cheng, Y., J. Ko et T. Lee (2016), « School autonomy, leadership and learning: A reconceptualisation », International Journal of Educational Management, vol. 30/2, http://dx.doi.org/10.1108/IJEM-08-2015-0108.

[4] Hanushek, E., S. Link et L. Woessmann (2012), « Does school autonomy make sense everywhere? Panel estimates from PISA », Economics Working Paper Series, n²96, Asian Development Bank.

[1] OCDE (2018), Regards surl'éducation 2018: Les indicateurs de l'OCDE, Éditions OCDE, Paris, http://dx.doi. org/10.1787/ eag-2018-fr.

[5] OCDE (2017), School Choice and School Vouchers: An OECD Perspective, OCDE, Paris, http://www.oecd.org/ education/School-choice-and-school-vouchers-an-OECD-perspective.pdf.

[9] OCDE (2016), Regards sur l'éducation 2016 : Les indicateurs de l'OCDE, Éditions OCDE, Paris, http://dx.doi. org/10.1787/eag-2016-fr.

[2] OCDE (2016), Résultats du PISA (Volume II) : Politiques et pratiques pour des établissements performants, PISA, Éditions OCDE, Paris, https://doi.org/10.1787/9789264267558-fr.

[3] OCDE (2014), Résultats de TALIS 2013 : Une perspective internationale sur l'enseignement et l'apprentissage, TALIS, Éditions OCDE, Paris, https://doi.org/10.1787/9789264214293-fr.

[10] OCDE (2011), "Autonomie et responsabilisation des établissements d'enseignement: Quel impact sur la performance des élèves? ", PISA à la loupe, n 9, Éditions OCDE, Paris, https://doi. org/10.1787/5k9h2r5nc2vb-fr.

[11] Pont, B., D. Nusche et H. Moorman (2008), Améliorer la direction des établissements scolaires, Volume 1 Politiques et pratiques, Éditions OCDE,, Paris, https://doi.org/10.1787/9789264044739-fr.

[6] Rodriguez, A. et K. Hovde (2002), « The challenge of school autonomy: Supporting principals », LCSHD Paper Series, n77, Bureau régional Amérique latine et Caraỉbes, Banque mondiale.

\begin{tabular}{|c|c|}
\hline VOIR & $\begin{array}{l}\text { Www.oecd.org/education/education-at-a-glance-19991487.htm } \\
\text { Indicateurs de l'éducation à la loupe (numéros précédents) } \\
\text { PISA à la loupe } \\
\text { L'enseignement à la loupe }\end{array}$ \\
\hline
\end{tabular}

PROCHAIN NUMÉRO Niveau de formation et résultats sur le marché du travail: comment se situent les adultes nés à l'étranger par rapport à leurs pairs nés dans le pays?

\section{9) ODE $\quad \begin{aligned} & \text { CONTACTER : } \\ & \text { Fatine Guedira (Fatine.Guedira@oecd.org) }\end{aligned}$}

Corinne Heckmann (Corinne.Heckmann@oecd.org)

Pauline Le Pape

Crédit photo : @ Christopher Futcher / iStock; @ Marc Romanelli / Gettyimages; @ michaeljung / Shutterstock; @ Pressmaster / Shutterstock Ce document est publié sous la responsabilité du Secrétaire général de l'OCDE. Les opinions qui y sont exprimées et les arguments qui y sont employés ne reflètent pas nécessairement les vues officielles des pays membres de l'OCDE.

Ce document, ainsi que les données et cartes qu'il peut comprendre, sont sans préjudice du statut de tout territoire, de la souveraineté s'exerçant sur ce dernier, du tracé des frontières et limites internationales, et du nom de tout territoire, ville ou région.

Les données statistiques concernant Israël sont fournies par et sous la responsabilité des autorités israéliennes compétentes. L'utilisation de ces données par l'OCDE est sans préjudice du statut des hauteurs du Golan, de Jérusalem-Est et des colonies de peuplement israéliennes en Cisjordanie aux termes du droit international. 